\title{
Effects of Subcytotoxic Exposure of Silver Nanoparticles on Osteogenic Differentiation of Human Bone Marrow Stem Cells
}

\author{
Alexander K. Nguyen, ${ }^{1,2}$ Reema Patel, ${ }^{2}$ Jade M. Noble, ${ }^{3}$ Jiwen Zheng, ${ }^{2}$ Roger J. Narayan, ${ }^{1}$ \\ Girish Kumar, ${ }^{2}$ and Peter L. Goering ${ }^{2}$
}

\begin{abstract}
Introduction: In vitro toxicology evaluations utilizing human stem cell models represent attractive alternatives to conventional animal models, which are not always predictive of human responses. Silver nanoparticles $(\mathrm{AgNP})$ are a potent antimicrobial for use in orthopedic devices. However, AgNP exposure may alter the behavior of stem cells within the bone marrow. The aim of this study was to determine if differences in cell proliferation, early, and late osteogenic differentiation markers can be detected in osteogenically differentiating human bone marrow mesenchymal stem cells (hBMSC) over a 21-day period at AgNP concentrations that are not considered cytotoxic after 24-hour exposure per ISO 10993-5 cytotoxicity testing guidelines.

Materials and Methods: Polyvinylpyrrolidone (PVP)-coated $10 \mathrm{~nm}$ AgNP were evaluated for their short-term (24-hour) cytotoxicity to hBMSC using the MTT assay to determine subcytotoxic concentrations for the subsequent long-term study investigating osteogenic differentiation. hBMSC were exposed to 1,5 , or $10 \mu \mathrm{g} / \mathrm{mL} \mathrm{AgNP}$ in three different exposure scenarios: single (24-hour), repeated (24-hour at 1, 7, and 14 days), or continuous (21day) exposure. Alkaline phosphatase (early osteogenic differentiation marker), hydroxyapatite deposition (late marker), and cell proliferation were measured at days 1, 7, 14, and 21.

Results: AgNP exposure reduced cell proliferation for all treatments. Neither differentiation marker expression was observed at any timepoint in $\mathrm{hBMSC}$ exposed to $\mathrm{AgNP}$ at $10 \mu \mathrm{g} / \mathrm{mL}$ for any exposure scenario or in cells exposed to $5 \mu \mathrm{g} / \mathrm{mL}$ in the repeated or continuous exposure scenarios.

Conclusions: Differences in proliferation and osteogenic marker expression were detected in the different exposure scenarios at AgNP concentrations identified as subcytotoxic in a 24-hour exposure assay.

Keywords: human bone marrow stem cell, hydroxyapatite, osteogenic differentiation, prolonged exposure, silver nanoparticle
\end{abstract}

\section{Introduction}

I NFECTIONS ASSOCIATED with medical devices such as orthopedic implants are a common complication, which often necessitates explantation of the device. Recently published statistics for total knee arthroplasty performed between 1999 and 2015 revealed infection in 1.0\%-1.5\% of all orthopedic surgeries and was a close-second cause of revision surgeries. ${ }^{1}$ Staphylococcus was found to be the leading organism in orthopedic surgeries at $29 \% .^{2}$ This also includes methicillin-resistant Staphylococcus aureus, which does not respond well to antibiotic treatment. Due to the increasing risk of antibiotic resistance, silver as an alternate mechanism for infection control would be advantageous. Incorporation of silver into medical devices is shown to reduce concentrations of various microbes, including $S$. aure$u s$, and inhibit the formation of biofilms. ${ }^{3,4}$ Both ionic and particulate forms of silver are used in skin-contacting medical devices, such as wound dressings ${ }^{5}$ or dermal reconstruction matrices, ${ }^{6}$ and multiple external-communicating

\footnotetext{
${ }^{1}$ University of North Carolina/North Carolina State University Joint Department of Biomedical Engineering, Raleigh, North Carolina.

${ }^{2}$ Office of Science and Engineering Laboratories, Center for Devices and Radiological Health, U.S. Food and Drug Administration, Silver Spring, Maryland.

${ }^{3}$ Office of Generic Drugs, Center for Drug Evaluation and Research, U.S. Food and Drug Administration, Silver Spring, Maryland.
} 
devices, such as Foley catheters containing silver are commercially available.

Despite the beneficial antimicrobial properties, silver could have adverse effects on human cells if released in sufficient doses to the systemic circulation. For example, uncoated $35 \mathrm{~nm}$ silver nanoparticles (AgNP) were found to exceed the 5\% hemolysis threshold in ASTM E2524-08 at $70 \mu \mathrm{g} / \mathrm{mL}$. $^{7} \mathrm{AgNP}$ (12.5 nm diameter) were also found to significantly alter several hematologic parameters at a $30 \mu \mathrm{g} / \mathrm{mL}$ threshold concentration in a blood loop model. ${ }^{8}$

Long-term silver exposure resulting from device implantation could raise the possibility of adverse local responses in the surrounding tissue. Stem cells in bone marrow play a role in a wide array of tissue regeneration tasks, such as the growth of new bone, fat, and blood cells. While impairment of any of these functions might negatively influence patient treatment outcomes, adverse effects on differentiation into the osteocyte lineage would slow or prevent osseointegration of the implant. This could lead to loosening, which is the leading cause of revision surgery. ${ }^{1}$

AgNP characteristics such as size, coating type, and surface chemistry influence biological outcomes such as cytotoxicity, nanoparticle uptake, and intracellular localization, ${ }^{9,10}$ and the change of any one of these parameters can have drastic effects. For example, equimolar concentrations of 3,10,50, and $100 \mathrm{~nm}$ diameter AgNP had different effects on zebrafish mortality and a number of sublethal effects such as malformation of different parts. ${ }^{11}$ Thus, the large number of possible combinations would be a challenge to test with conventional animal models. An alternative in vitro model using human stems cells and allowing for long-term exposure to the toxicant of interest would not only be more relevant to human biological and toxicological responses but also be more practical to evaluate a large number of potential toxicants.

Therefore, the objective of this study was to assess the effect of prolonged AgNP exposure on osteogenic differentiation of human bone marrow mesenchymal stem cells (hBMSC) at subcytotoxic AgNP concentrations. Additionally, different exposure scenarios up to 21 days were investigated to model various degrees of duration (e.g., acute, moderate, and prolonged) of implant tissue contact. Thus, the hypothesis that differences in cell proliferation, early, and late osteogenic differentiation markers can be detected in differentiating hBMSC over a 21-day period at AgNP concentrations that are not considered cytotoxic after 24-hour exposure per ISO 10993-5 cytotoxicity testing guidelines was tested. Findings from this in vitro model can help inform further understanding of how human stem cells respond to subcytotoxic AgNP concentrations for extended exposures with additional benefits of (1) reducing the necessity of some animal testing and (2) contributing to understanding risks and benefits of this material for medical device implant applications.

\section{Materials and Methods}

hBMSC (PCS-500-012; ATCC, Manassas, VA) were cultured in mesenchymal stem cell basal medium (PCS-500030; ATCC) supplemented with the mesenchymal stem cell growth kit (PCS-500-041; ATCC) and incubated at $37^{\circ} \mathrm{C}$, $5.0 \% \mathrm{CO}_{2}$, and $90 \%$ relative humidity. Osteogenic differenti- ation was performed using the Osteocyte Differentiation Tool (PCS-500-052; ATCC) in place of the medium. The mesenchymal stem cell medium and Osteogenic Differentation Tool are refered to as osteogenic supplement negative (OS-) and positive (OS+) respectively in this study. Other reagents included Trypsin-EDTA (9002-07-7; Sigma-Aldrich, St. Louis, MO), Dulbecco's phosphate-buffered saline (PBS; 14190-xxx; Thermo Scientific, Waltham, MA), Triton X-100 (SigmaAldrich), 37\% formaldehyde (252549; Sigma-Aldrich), and ultrapure water from a NANOpure Diamond water purifier (APS Water Services Corp., Van Nuys, CA).

\section{AgNP characterization}

Polyvinylpyrrolidone (PVP)-coated, $10 \mathrm{~nm}$ diameter AgNP (BioPure; NanoComposix, San Diego, CA) were used in this study. AgNP stock solutions were received as a $1 \mathrm{mg} / \mathrm{mL}$ solution in water and were characterized using a JEM $2010 \mathrm{~F}$ (JEOL, Tokyo, Japan) transmission electron microscope (TEM). AgNP were also diluted in cell culture medium immediately before application to the cryo-TEM grids and the cells per experimental design.

Dynamic light scattering analysis was performed with a Zetasizer Nano ZS (Malvern Panalytical, Malvern, United Kingdom). Hydrodynamic diameter measurements were performed on $20 \mu \mathrm{g} / \mathrm{mL} \mathrm{AgNP}$ in $0.02 \mu \mathrm{m}$ filtered ultrapure water (MilliporeSigma, Burlington, MA). Zeta potential measurements were performed on $100 \mu \mathrm{g} / \mathrm{mL} \mathrm{AgNP}$ in $10 \mathrm{mM} \mathrm{NaCl}$ inside a DTS1070-folded capillary cell (Malvern Panalytical).

Cryo-TEM was performed on a JEOL 1400 (JEOL USA, Inc., Acworth, GA) operated at $80 \mathrm{kV}$ and equipped with an Orius SC1000 CCD camera (Gatan, Pleasanton, CA). Aliquots $(5 \mu \mathrm{L})$ of $100 \mu \mathrm{g} / \mathrm{mL}$ AgNP in ultrapure water, OSmedia, or OS+ media were pipetted onto glow discharged copper Quantifoil (Electron Microscopy Sciences, Hatfield, PA) grids and plunge-frozen using a Leica EM GP automatic plunge freezer (Leica Microsystems, Wetzlar, Germany). Images were binned by 1 , resulting in pixel sizes of $0.4 \mathrm{~nm}$.

\section{Cell culture}

For each experiment replicate, $1 \times 10^{6} \mathrm{hBMSC}$ stored in liquid nitrogen at passage 4 were thawed and seeded into a T-150 flask and incubated overnight. A media change was performed the following day to remove the dimethylsulfoxide cryopreservative and dead/unattached cells and then allowed to divide for $\sim 1$ week to reach $80 \%$ confluence. Cells were trypsinized, centrifuged at $200 \mathrm{~g}$ for 5 minutes, and resuspended in media. The hBMSC suspension cell density was measured with a hemocytometer and diluted to $5.0 \times 10^{4}$ cells $/ \mathrm{mL}$ for seeding. Cells used in both the $24-$ hour exposure and 21-day culture procedures were therefore used at passage 5; passage 6 is the highest passage recommended by ATCC for osteogenic differentiation with the Osteocyte Differentiation Tool.

\section{Viability of $h B M S C$ after a single 24-hour exposure to AgNP}

Cytotoxicity of AgNP in a single 24-hour exposure was measured via the MTT assay (CellTiter 96 Non-radioactive Cell Proliferation Assay, G4000; Promega, Madison, WI) 
and read with an OptiMax plate reader (Molecular Devices, Sunnyvale, CA). Three wells per treatment in a 96-well plate were seeded with $1.0 \times 10^{4}$ cells per well in $200 \mu \mathrm{L}$ medium and allowed to attach over 24 hours. Cells were then exposed to $1,5,10,25$, or $50 \mu \mathrm{g} / \mathrm{mL}$ AgNP for 24 hours in either OSor OS+ media. Medium alone and medium with $3 \mathrm{mM}$ $\mathrm{AgNO}_{3}$ were used as negative and positive controls, respectively. Cell-free wells of the corresponding solution were used as the background. An aliquot of $15 \mu \mathrm{L}$ MTT dye was added to each well and allowed to metabolize for 3 hours. The kit surfactant solution was used to stop the reaction and dissolve the colored metabolite. The plates were read after 30 minutes of incubation and 30 minutes on a shake plate. For each well, absorbance at $650 \mathrm{~nm}$ was subtracted from the absorbance at $570 \mathrm{~nm}$. Viability is calculated as a percent of the negative control and reported as the means and standard deviations (SD) of three independent replicate experiments.

\section{Long-term hBMSC culture using three dosing/exposure scenarios}

Three long-term subcytotoxic exposure scenario studies involved monitoring hBMSC with a combination of the following treatment conditions: (1) exposure to one of the three AgNP dosing/exposure scenarios, (2) exposure to one of the three different AgNP concentrations, and (3) culture in media with or without osteogenic supplements. The exposure scenarios are summarized in Figure 1: a single 24-hour exposure (single), three 24-hour exposures performed on days 1, 7, and 14 (repeated), and continuous exposure to AgNP for 21 days with AgNP refreshed $3 \times$ per week (continuous). AgNP concentrations of 1,5 , or $10 \mu \mathrm{g} / \mathrm{mL}$ in media with $(\mathrm{OS}+)$ or without $(\mathrm{OS}-)$ osteogenic supplements were prepared immediately before exposure to the cells.

For each of four sampling timepoints $(1,7,14$, and 21 days), six 96-well plates were used corresponding to one of the three exposure conditions, and the use of media with or without osteogenic supplements. On each plate, two wells per AgNP concentration $(1,5$, or $10 \mu \mathrm{g} / \mathrm{mL})$ for each of the planned assays were seeded with $1.0 \times 10^{4}$ cells per well in $200 \mu \mathrm{L}$ medium and incubated for 24 hours before starting the prescribed exposure scenario; $\mathrm{hBMSC}$ at the day $1 \mathrm{AgNP}$ exposure was $\sim 50 \%$ confluent. hBMSC grown with or without osteogenic supplements and in the absence of AgNP

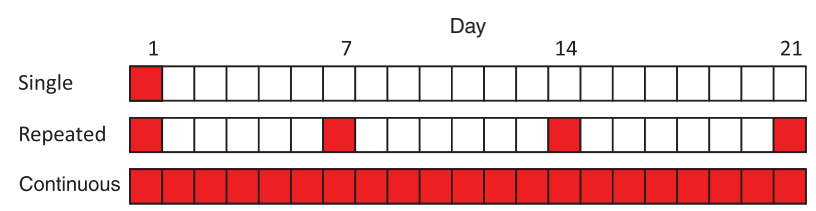

FIG. 1. Schedule of AgNP dosing of hBMSC cultures for three separate exposure scenarios: single 24-hour exposure, repeated weekly 24-hour exposures, or continuous exposure. Each square represents a 24-hour period with red squares indicating periods of AgNP exposure. Cell proliferation, alkaline phosphatase expression, and hydroxyapatite content were measured on days $1,7,14$, and 21 for all exposure scenarios. Fluorescence imaging of hydroxyapatite was performed on day 21 only. hBMSC, human bone marrow mesenchymal stem cells. Color images are available online. were used as the negative controls. Data from the negative controls are shared between all exposure scenarios but are included on all subcharts in Figures 4-7 for ease of comparison. Media were changed three times a week for the 21day culture period with either media or media with AgNP applied to the cells according to the relevant exposure scenario (Fig. 1). If a well in the single or repeated exposure scenarios received media with AgNP, the particles were removed 24 hours later and replaced with the corresponding media.

For each timepoint, plates were processed for the different assays: Quant-iT PicoGreen, Alkaline Phosphatase (ALP) Liquicolor, and OsteoImage Mineralization assays. ALP was measured immediately at each timepoint, but PicoGreen and OsteoImage assays were performed in bulk after all plates had been harvested. In preparation for storage, media were aspirated from all wells; OsteoImage wells were fixed with $3.7 \%$ formaldehyde in PBS for 30 minutes, rinsed $2 \times$ with PBS, and then, the PBS aspirated before storage at $-80^{\circ} \mathrm{C}$.

PicoGreen assay. Cells designated for the PicoGreen assay (Quant-iT PicoGreen dsDNA assay kit, P7589; Thermo Scientific) were previously frozen at $-80^{\circ} \mathrm{C}$ at each timepoint so were thawed to room temperature before digestion with a papain suspension (lot 32J13550, LS003127; Worthington, Lakewood, NJ). Papain was dissolved at a $0.21 \mathrm{U} / \mathrm{mL}$ concentration in $1.75 \mathrm{mg} / \mathrm{mL}$ L-cysteine in PBS, and $200 \mu \mathrm{L}$ of this solution was added to each well. The entire plate was sealed with aluminum sealing tape and heated at $60^{\circ} \mathrm{C}$ for 18 hours. After digestion, the plates were cooled to room temperature over 1 hour. From each well, three aliquots of $50 \mu \mathrm{L}$ were transferred to three wells on a separate 96-well plate for measurement in triplicate. A double-stranded DNA concentration series $(0,40,100,200,400,600,1000,1400$, 1600 , and $2000 \mathrm{ng} / \mathrm{mL}$ ) was prepared by diluting the kit dsDNA stock solution in Tris-EDTA buffer. For each dsDNA ladder concentration, $50 \mu \mathrm{L}$ was added to two wells for measurement in duplicate. PicoGreen dye in TrisEDTA buffer was mixed according to the manufacturer's specifications, and $50 \mu \mathrm{L}$ was added to all wells and the samples stained for 5-10 minutes. The plate was read in a fluorescent plate reader using $485 \mathrm{~nm}$ excitation and $538 \mathrm{~nm}$ emission wavelengths. For each set of three measurement wells corresponding to a single well on the initial plate, the average fluorescence intensity was fit to the DNA standard curve and multiplied by the $200-\mu \mathrm{L}$ volume used in the papain digestion to calculate the DNA mass within the well.

ALP assay. ALP was measured using the Alkaline Phosphatase Liquicolor kit (2900-430; Stanbio, Boerne, TX) using SER-T-FY 1 Level 1 Control Serum (lot 151631, G427-86; Stanbio) for controls. Kinetic measurements for this assay were performed on a SpectraMax 190 plate reader (Molecular Devices). Media from ALP assay wells were aspirated and replaced with $100 \mu \mathrm{L} 0.2 \%$ Triton X-100 in PBS for 20 minutes. A new 96-well plate was prepared where three wells each were allocated for negative and positive controls: $0.2 \%$ Triton $\mathrm{X}-100,5 \mu \mathrm{L}$ serum, $15 \mu \mathrm{L}$ serum, and $35 \mu \mathrm{L}$ serum. These control wells were diluted to $75 \mu \mathrm{L}$ total volume with additional $0.2 \%$ Triton X-100. The permeabilized cells were diluted with an additional $100 \mu \mathrm{L}$ of $0.2 \%$ Triton $\mathrm{X}-100$ and mixed thoroughly. Two $75 \mu \mathrm{L}$ aliquots from each of 
these solutions were added to two wells on the new plate for measurement in duplicate. A $75 \mu \mathrm{L}$ aliquot of the kit working reagent was added to each well; the plate was then immediately placed into a fluorescent plate reader for kinetic measurements. Absorbance at $405 \mathrm{~nm}$ was read once per minute over 5 hours. The rate of generation of the colored compound is initially linear and proportional to the ALP content. For each well, the linear region of the curve was determined between the start of data collection and the timepoint when the $R^{2}$ value of the linear regression was greater than 0.98 . The slope of the linear region was compared with the slope of the serum control wells to calculate the ALP concentration.

Osteolmage fluorescent microscopy/mineralization quantification assay. Mineralization quantification was performed with the OsteoImage Mineralization Assay (PA-1503; Lonza, Walkersville, MD) that specifically stains hydroxyapatite; fluorescence quantification using a plate reader and fluorescence microscopy was performed in the same well. The hBMSC were previously fixed in $3.7 \%$ formaldehyde and stored frozen at $-80^{\circ} \mathrm{C}$. OsteoImage dye was diluted 1:100 in the supplied wash buffer per the manufacturer's instructions; $100 \mu \mathrm{L}$ was added to each well, the samples stained for 30 minutes, and then washed $3 \times$ with the wash buffer. The hydroxyapatite fluorescence signal was read at $492 \mathrm{~nm}$ excitation and $520 \mathrm{~nm}$ emission wavelengths. Fluorescent images of the wells after 21 days in culture were obtained using an Eclipse TE2000-U fluorescence microscope (Nikon, Tokyo, Japan).

\section{Statistical analysis}

Statistical analysis and graphing were performed using GraphPad Prism 6 (San Diego, CA). Cytotoxicity after 24hour AgNP exposure was analyzed by multiple Student's $t$-tests comparing each treatment with the negative control. Proliferation during the 21-day study measured by the PicoGreen DNA assay was analyzed using two-way analysis of var- iance between AgNP concentrations and exposure scenarios within each timepoint; statistical significance was determined using the Tukey post hoc analysis $(p<0.05)$. Detection of ALP and mineralization, early and late markers of osteogenic differentiation, respectively, was analyzed by comparing OS+ supplemented cells with the corresponding OS - treatment for each timepoint using a one-tailed Student's $t$-test. Since both ALP expression and hydroxyapatite deposition were near baseline levels for all OS - cells, data were summarized as the difference between the OS+ and OS- supplemented groups for clarity. Thus, the resulting means and SDs were calculated using $\bar{X}_{\mathrm{OS}+}-\bar{X}_{\mathrm{OS}-} \pm \sqrt{\mathrm{SD}_{\mathrm{OS}+}{ }^{2}+\mathrm{SD}_{\mathrm{OS}-}{ }^{2}}$.

\section{Results}

\section{AgNP characterization}

Particles $(n=140)$ in one TEM image (Fig. 2) were analyzed using the ImageJ (NIH, Bethesda, MD). Particle diameter averaged $9.95 \mathrm{~nm}$ with a SD of $2.22 \mathrm{~nm}$. The morphology was approximately circular with average major and minor elliptical axes of 10.52 and $9.44 \mathrm{~nm}$, respectively. AgNP Z-average hydrodynamic diameter was measured to be $18.33 \mathrm{~nm}$, with a SD of $0.73 \mathrm{~nm}$ over six measurements. Zeta potential was measured to be $-15.6 \mathrm{mV}$, with a SD of $0.95 \mathrm{mV}$ over four measurements.

Cryo-TEM (Fig. 2) was performed on AgNP in both OSand OS+ media to image the state of AgNP that the hBMSC were exposed to. AgNP dispersed in water was found to be monodisperse. In both media types, AgNP appeared as single particles or as small agglomerates of less than 10 particles.

\section{Dose-finding for cytotoxicity of AgNP after a single 24-hour exposure}

An AgNP concentration-dependent decrease in hBMSC viability was observed using the MTT assay (Fig. 3). Based on the international standard ISO 10993-5, ${ }^{12}$ a chemical is noted to have cytotoxic potential if the viability falls
FIG. 2. Representative TEM image (top left) of $10 \mathrm{~nm}$ PVP-coated AgNP. AgNP core diameter was determined to be $9.95 \mathrm{~nm}$ with a $2.22 \mathrm{~nm}$ SD by image analysis of $n=140$ particles. CryoTEM images of AgNP diluted in water (top right), OS- media (bottom left), and OS+ media (bottom right). AgNP, silver nanoparticles; SD, standard deviation; TEM, transmission electron microscope.

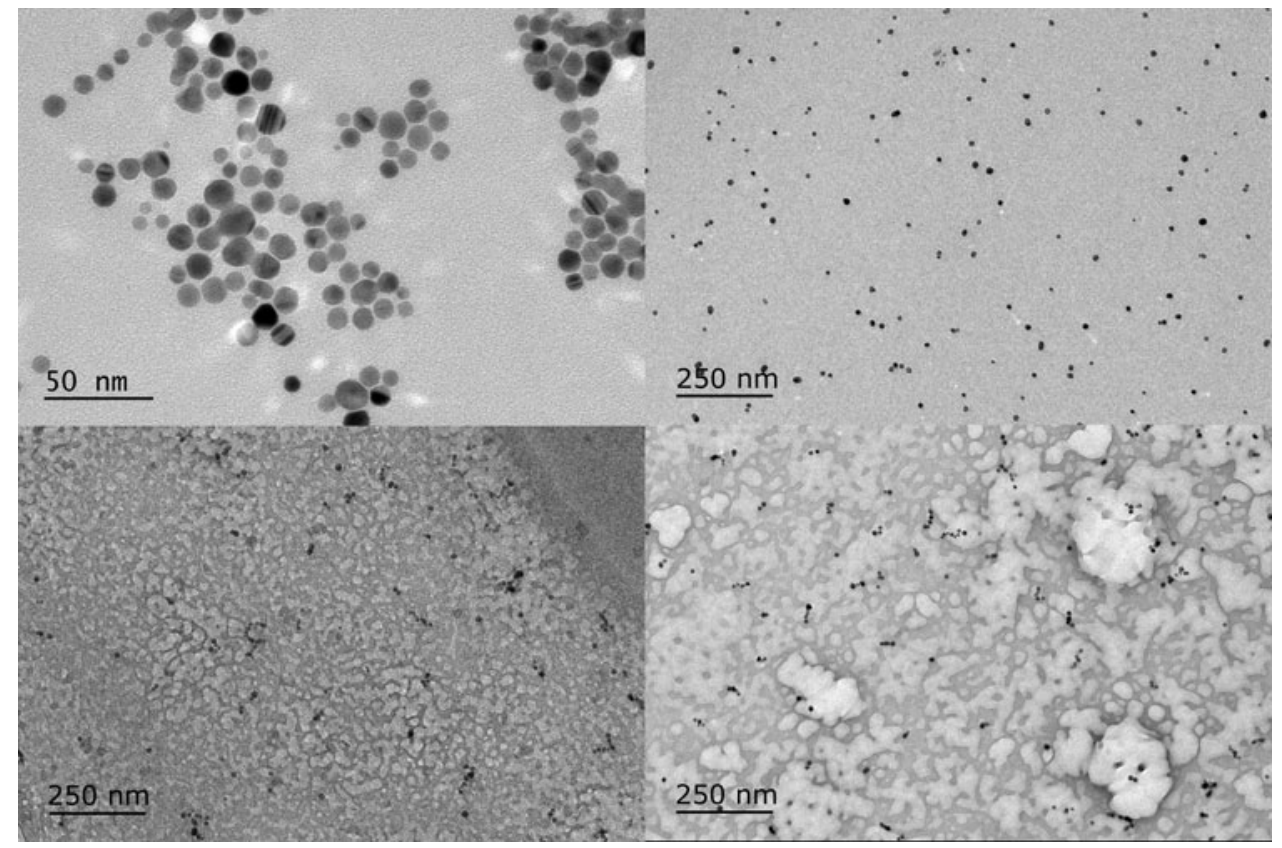




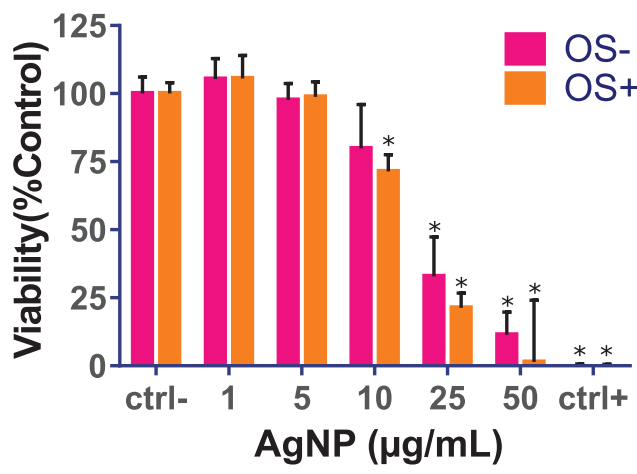

FIG. 3. Viability of hBMSC (MTT assay) cultured for 24 hours in the absence (OS-) or presence (OS+) of osteogenic supplements. Cells were exposed to various concentrations of $10 \mathrm{~nm}$ PVP-coated AgNP. Cells grown in media alone or media with $3 \mathrm{mmol} / \mathrm{L} \mathrm{AgNO}_{3}$ were used as the negative (ctrl-) and positive (ctrl+) controls, respectively. Values represent $\bar{x} \pm S D$ ( $n=3$ replicate experiments). Bars with asterisks are significantly different from the negative control $(p<0.05)$. SD, standard deviation. Color images are available online.

below $70 \%$. The viability of hBMSC exposed to 1 or $5 \mu \mathrm{g} / \mathrm{mL} \mathrm{AgNP}$ for 24 hours was not statistically different from the negative control. hBMSC exposed to $10 \mu \mathrm{g} / \mathrm{mL}$ AgNP exhibited viabilities of $80 \%$ and $71 \%$ of control when cultured in the absence and presence of osteogenic supplements, respectively. Concentrations of 25 and $50 \mu \mathrm{g} / \mathrm{mL}$ resulted in statistically significant decreases in viability, and the values were below the $70 \%$ cytotoxicity threshold. Therefore, concentrations of 1 and $5 \mu \mathrm{g} / \mathrm{mL}$ were included in the 21-day study as concentrations with no detected 24 hours of cytotoxicity, and $10 \mu \mathrm{g} / \mathrm{mL}$ AgNP included as the concentration that meets the criteria for nonsignificant cytotoxic potential according to the ISO 10993-5 standard.

\section{Cell proliferation}

Proliferation was assessed with the PicoGreen assay in which dsDNA concentrations can be quantitatively measured. The dsDNA concentration is proportional to the number of live cells remaining in the culture; dead cells detach from the plate and are removed with regular media changes. For easier data visualization, proliferation data were organized in two graphs; as a time course (Fig. 4) and individually by timepoint to more clearly show the dose-response and statistically significant differences (Fig. 5).

The behavior of undifferentiated and differentiating hBMSC is observed when considering the proliferation data as a time course (Fig. 4). Long-term cell culture of hBMSC without osteogenic supplements (OS-) was supported over the 21-day study with increasing proliferation observed at all timepoints under control conditions, which is as expected for a stem cell population. This increasing proliferation was also observed after single exposure to $1 \mu \mathrm{g} / \mathrm{mL}$ AgNP. No change in proliferation was observed after single exposure to $10 \mu \mathrm{g} / \mathrm{mL}$, single or repeated exposure to $5 \mu \mathrm{g} / \mathrm{mL}$, or with repeated exposure to $1 \mu \mathrm{g} / \mathrm{mL}$ AgNP. In contrast, hBMSC exposed to osteogenic supplements (OS+) under control conditions proliferated between day 1 and 7 after which the amount of dsDNA decreased at day 14 and 21. In general, differentiated cells do not proliferate; as such, the decrease in dsDNA mass was expected for a differentiated culture. Cell proliferation under all exposure conditions of 1 or
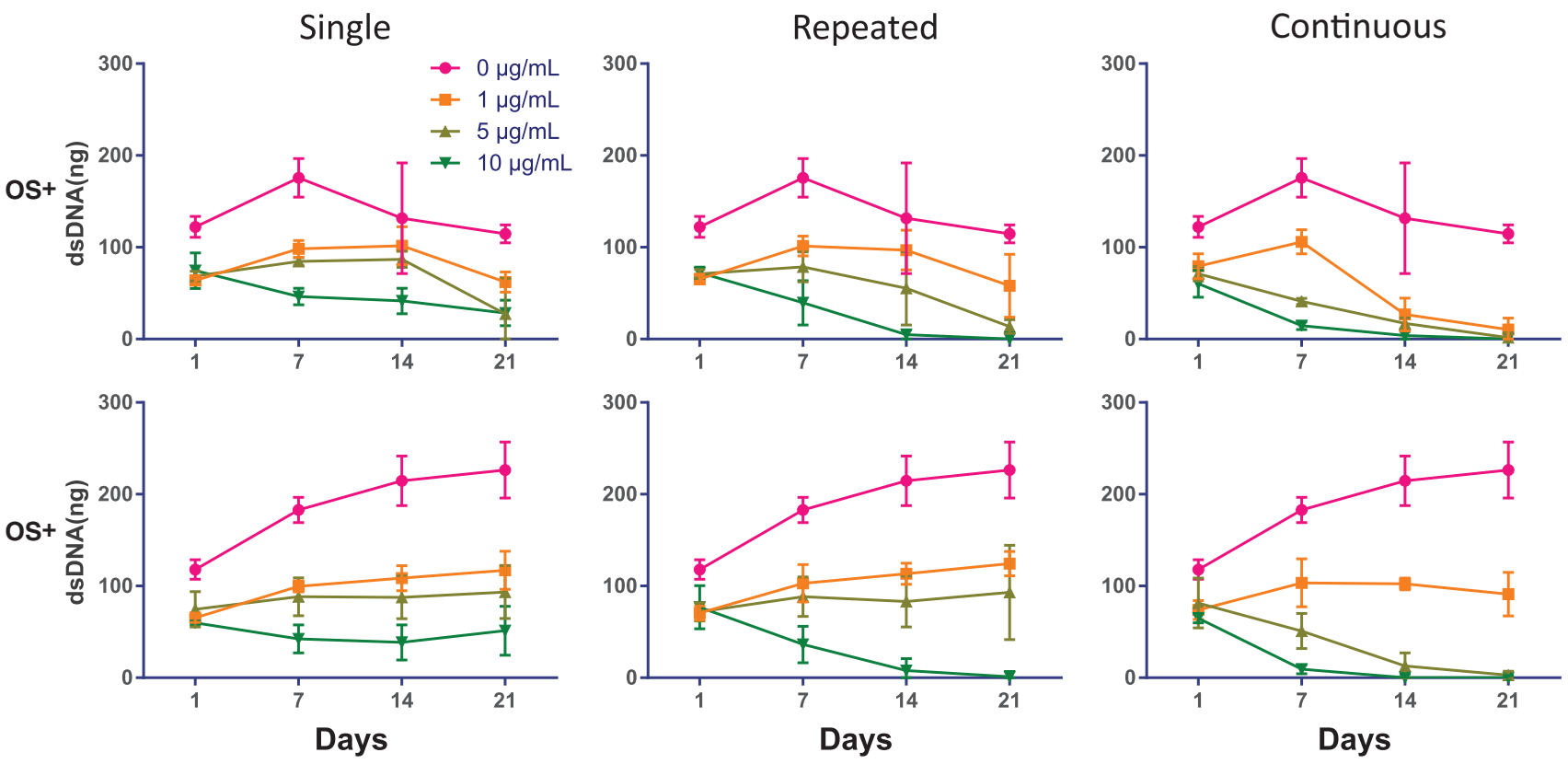

FIG. 4. Time-course responses for the proliferation of hBMSC (PicoGreen dsDNA assay) cultured in the absence (OS-) or presence (OS+) of osteogenic supplements under single, repeated, or continuous exposure (Fig. 1$)$ to 1,5 , or $10 \mu \mathrm{g} / \mathrm{mL}$ of $10 \mathrm{~nm}$ PVP-coated AgNP. Note that hBMSC not exposed to OS (OS-) or AgNP proliferated over the entire 21-day period, whereas the proliferation of hBMSC exposed to OS (OS+) peaked around day 7 and then declined. Values represent $\bar{x} \pm S D$ ( $n=3$ replicate experiments). Statistical analysis of these data is displayed in Figure 5. Color images are available online. 

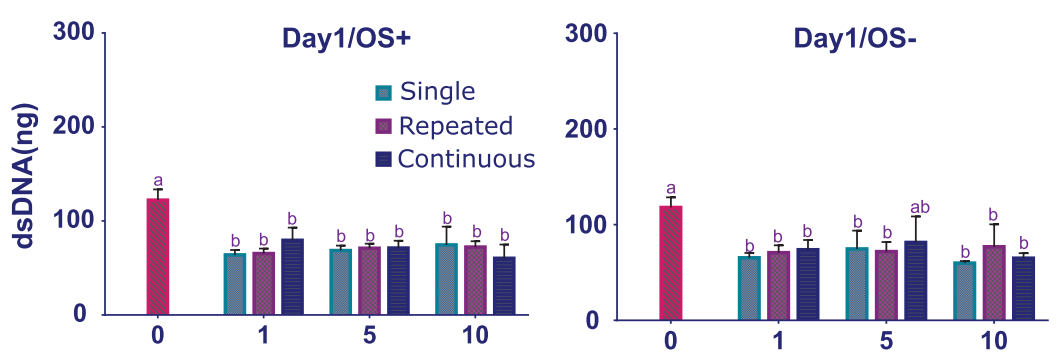

FIG. 5. Cell proliferation data from

Figure 4 plotted separately for days 1, 7, 14,
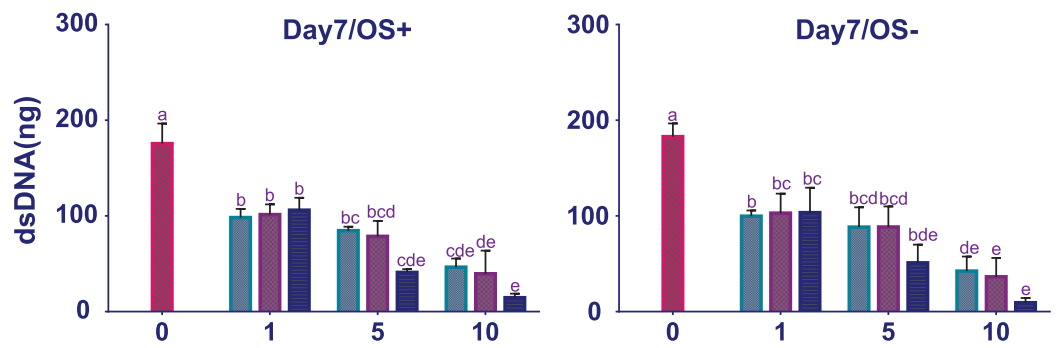
tistical differences of exposure (single, repeated, continuous) scenarios and AgNP dose. Values represent $\bar{x} \pm S D(n=3)$. Bars with no matching letters are statistically different $(p<0.05)$. Color images are available online.
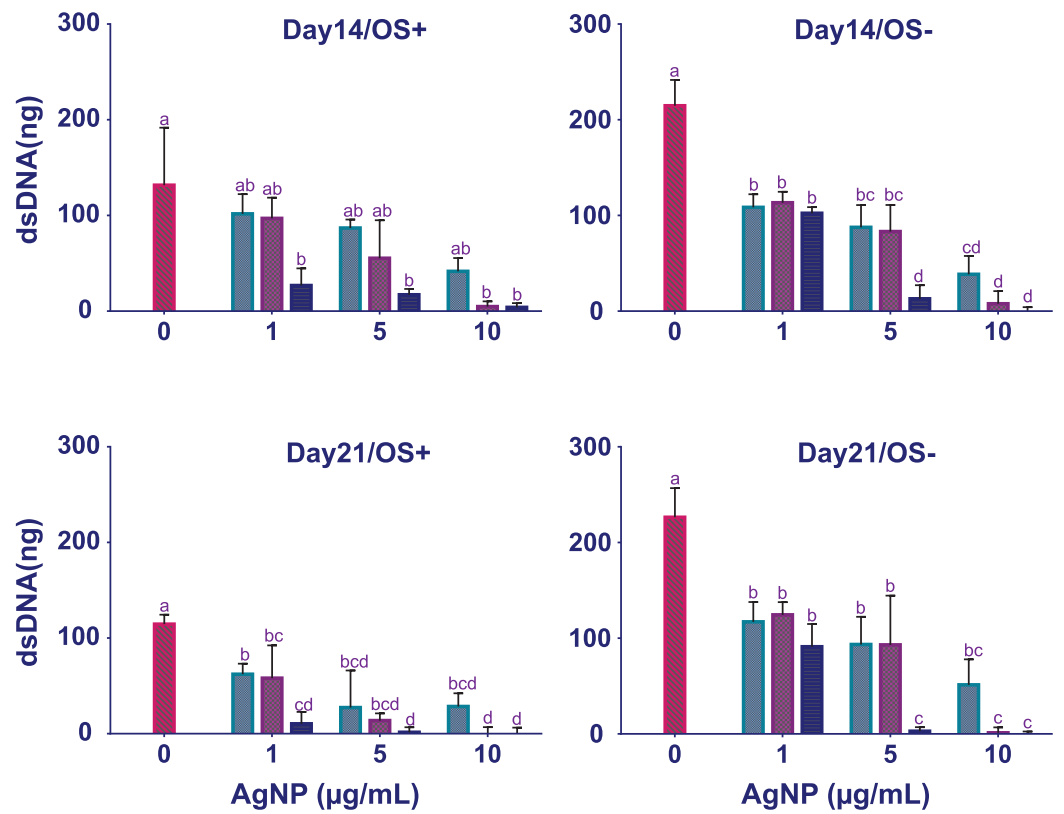

$5 \mu \mathrm{g} / \mathrm{mL}$ AgNP responded similarly as the control with high proliferation apparent at day 7. Proliferation under continuous exposure to $1 \mu \mathrm{g} / \mathrm{mL}$ AgNP also shows some proliferation at day 7; however, no increase in proliferation is observed at single or repeated exposure at $10 \mu \mathrm{g} / \mathrm{mL}$ or continuous exposure at 5 or $10 \mu \mathrm{g} / \mathrm{mL}$.

At day 1, no statistically significant difference in dsDNA content was found between any AgNP concentration or exposure scenario (Fig. 5). By day 7, evidence of a dose-related response was becoming evident, with proliferation in the $1 \mu \mathrm{g} / \mathrm{mL}$ AgNP treatment group being significantly higher than that in the $10 \mu \mathrm{g} / \mathrm{mL}$ group for both the OS+ supplemented and OS - cells. This dose-response was also observed at day 14 and 21 within each exposure scenario. Proliferation resulting from continuous exposure tended to be lower at all AgNP concentrations than the single or repeated exposure scenarios, although the differences between exposure scenarios using the same AgNP concentration were not statistically significant.

\section{Assessment of early and late osteogenic differentiation markers}

ALP expression as an early marker of osteogenic differentiation and hydroxyapatite content as a late marker of differentiation are displayed in Figures 6 and 7, respectively. In both assays, the intensity of the signal should only be used to detect whether osteogenic differentiation was taking place at a certain timepoint. This is due to the coarse nature of the timepoints preventing precise measurement of when 

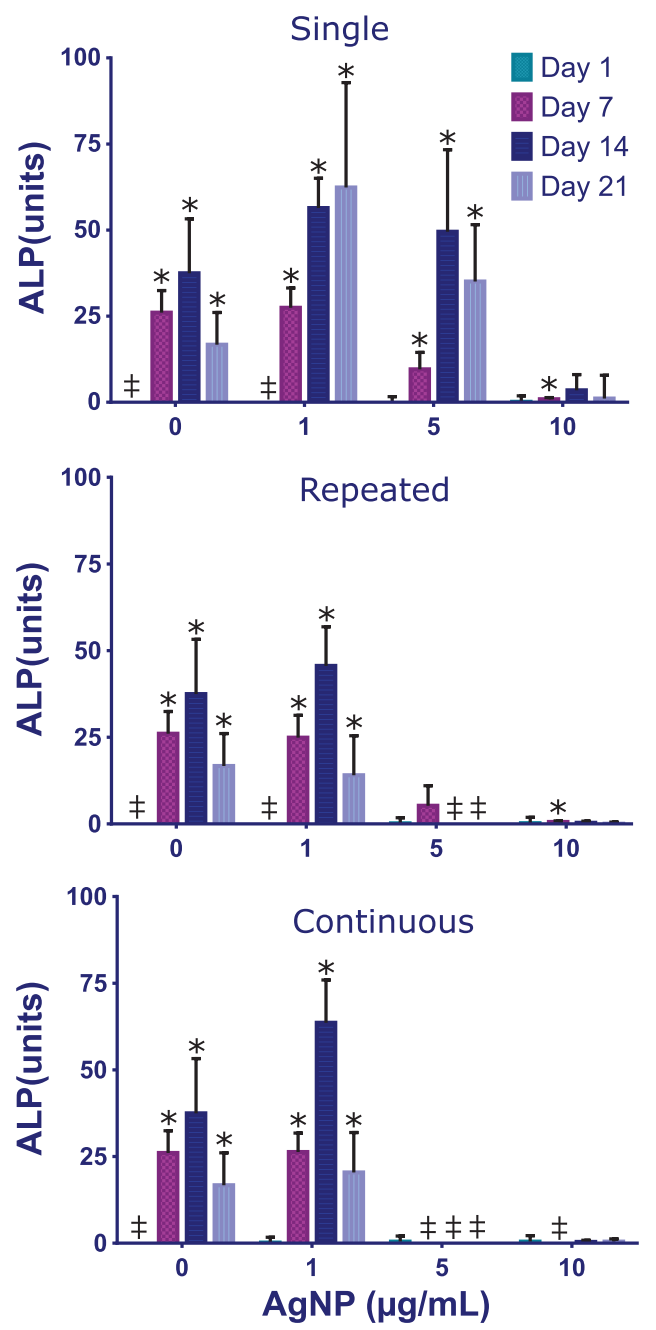

FIG. 6. Differences in ALP expression in hBMSC cultured in the presence $(\mathrm{OS}+)$ and absence of $(\mathrm{OS}-$ ) of osteogenic supplements under single, repeated, or continuous exposure (Fig. 1) to 1,5 , or $10 \mu \mathrm{g} / \mathrm{mL}$ of $10 \mathrm{~nm}$ PVP-coated AgNP. Elevated ALP expression is an early marker of osteogenic differentiation. Values plotted were derived by subtracting the signal values from OS - cells from the signals from OS+ supplemented cells. Therefore, the values represent $\bar{x} \pm S D$ calculated as $\bar{X}_{O S+}-\bar{X}_{O S-} \pm \sqrt{S D_{O S+}{ }^{2}+S D_{O S-}{ }^{2}}$ for $n=3$ replicate experiments. Bars with asterisks denote a statistically significant $(p<0.05)$ increase in ALP expression of OS+ supplemented cells over the corresponding OS - cells. Absent bars marked with $t$ are the result of negative values due to subtracting the signal values from OS- cells from near-baseline signals from OS+ supplemented cells. ALP, alkaline phosphatase. Color images are available online.

the signal is first observed. For example, ALP expression should increase until around day 14 and decrease afterward, but the exact time of the peak is unknown; comparison of one treatment group at the ALP expression peak and a second that had expressed it slightly earlier and has a smaller signal would yield false conclusions. Therefore, the ALP or hydroxyapatite signal for the OS+ supplemented cells were only compared with the corresponding OS - group for statistical analysis. ALP and hydroxyapatite signals were also near the baseline for all cells not exposed to osteogenic supple-
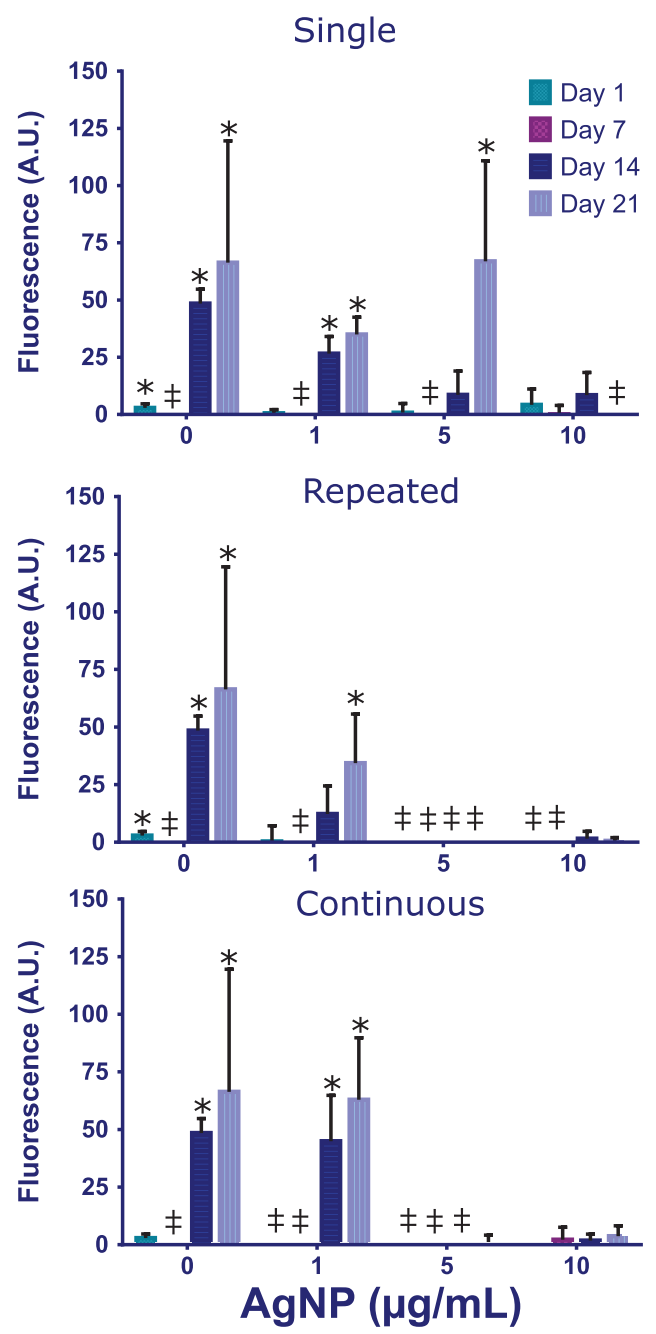

FIG. 7. Differences in hydroxyapatite content (OsteoImage Mineralization Assay) between TCPS with hBMSC cultured in the presence of osteogenic supplements (OS+) subtracted from TCPS with hBMSC cultured in the absence of osteogenic supplements (OS-) under single, repeated, or continuous exposure to 1,5 , or $10 \mu \mathrm{g} / \mathrm{mL}$ of $10 \mathrm{~nm}$ PVP-coated AgNP. Mineralization of the substrate is a late marker of osteogenic differentiation. Values plotted were derived by subtracting the signal values from OS- cells from the signals from OS+ supplemented cells. Therefore, the values plotted represent $\bar{x} \pm S D$ calculated as $\bar{X}_{O S+}-\bar{X}_{O S-} \pm \sqrt{S D_{O S+}{ }^{2}+S D_{O S-}{ }^{2}}$ for $n=3$ replicate experiments. Bars with asterisks denote a significant $(p<0.05)$ increase in mineralization of the substrate from OS+ supplemented cells over the corresponding $\mathrm{OS}-$ cells. Absent bars marked with $\ddagger$ are the result of negative values due to subtracting signal values from OS - cells from near-baseline signal values from OS+ supplemented cells. TCPS, tissue culture polystyrene surfaces. Color images are available online.

ments, so the data were displayed as the difference between the two groups for clarity.

In hBMSC cultured in the presence of osteogenic supplements under control conditions, ALP expression reached a peak after 14 days in culture with detectable increases by day 7 (Fig. 6). hBMSC exposed to $1 \mu \mathrm{g} / \mathrm{mL}$ in all exposure scenarios or exposed to a single exposure to $5 \mu \mathrm{g} / \mathrm{mL}$ had 

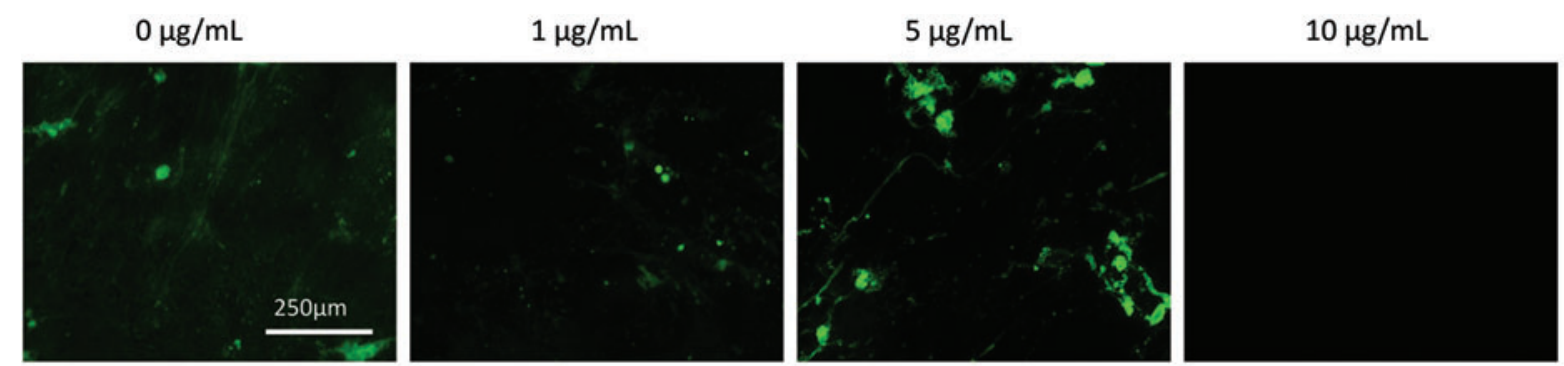

Single
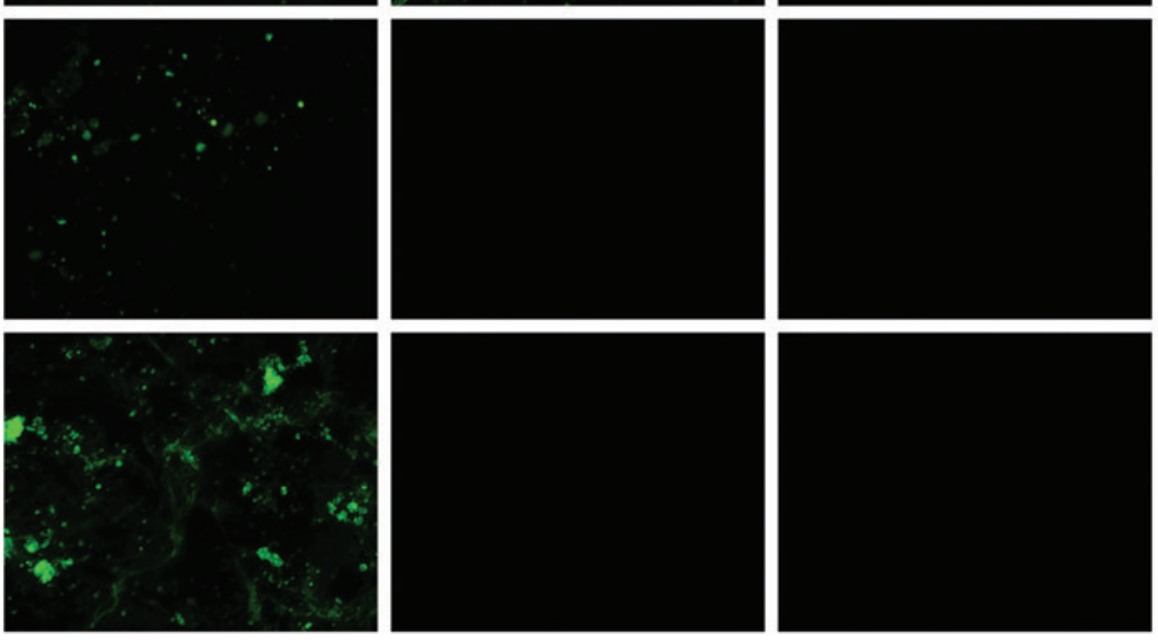

Repeated

Continuous

FIG. 8. Fluorescence microscopy images corresponding to the OsteoImage mineralization data summarized in Figure 7 for OS+ supplemented hBMSC cultured for 21 days and exposed to AgNP under single, repeated, or continuous exposure scenarios. No hydroxyapatite was detected with repeated or continuous exposure to $5 \mu \mathrm{g} / \mathrm{mL}$ AgNP or for any exposure to $10 \mu \mathrm{g} / \mathrm{mL}$ AgNP. Color images are available online.

significantly increased ALP levels at day 7 and expressed detectable levels of hydroxyapatite at day 21 (Fig. 7). Of these, all treatment groups except for single exposure to $5 \mu \mathrm{g} / \mathrm{mL}$ AgNP and repeated exposure to $1 \mu \mathrm{g} / \mathrm{mL}$ had detectable hydroxyapatite at day 14 ; the corresponding fluorescence images of mineralized substrates after 21 days in culture are displayed in Figure 8. Thus, detection of ALP at day 7 is highly predictive of eventual mineralization of the substrate.

\section{Discussion}

The broad antimicrobial properties of AgNP make them attractive for use in medical device materials; however, these benefits must be balanced with the potential effects on surrounding tissue. In this study, physiologically relevant AgNP concentrations were first evaluated for their cytotoxicity after 24-hour exposure. Sussman et al. investigated ionic $\mathrm{Ag}^{+}$and AgNP content in multiple commercially available medical devices by extracting the devices in multiple solvents, including citrated human plasma. The total silver concentration was $\sim 300 \mu \mathrm{g} / \mathrm{mL}$ in water extract (using a $3 \mathrm{~mL} / \mathrm{cm}^{2}$ extraction ratio) or $\sim 10 \mu \mathrm{g} / \mathrm{mL}$ in human plasma. ${ }^{13}$ AgNP in these products were bound to the device surfaces; therefore, the $1-50 \mu \mathrm{g} / \mathrm{mL}$ range used in the 24 -hour exposure in the present study would represent an appropriate concentration range approximating AgNP eluted from medical device surfaces. No cytotoxicity was detected in hBMSC in either medium (OS+ and $\mathrm{OS}-$ ) after 24 hours of exposure to 1 or $5 \mu \mathrm{g} / \mathrm{mL} \mathrm{AgNP}$. As for the $10 \mu \mathrm{g} / \mathrm{mL}$ concentration, traditional cytotoxicity testing as described in the ISO 10993 Part 5 standard $^{12}$ specifies that a $70 \%$ viability after 24 hours of exposure is considered as having "cytotoxic potential"; the average viability of hBMSC exposed to $10 \mu \mathrm{g} / \mathrm{mL}$ in either media was above this threshold and was therefore included in the 21-day study.

The mechanism of AgNP cytotoxicity is an active area of research with particle parameters such as diameter, surface charge, geometry, and ion dissolution playing a role. $9,10,14$ Thus, one would expect different degrees of cytotoxicity responses when considering particles of different diameters and/or coatings. For example, Sengstock et al. reported no observable change in hBMSC viability in $50 \mathrm{~nm}$ PVP-coated AgNP over 24 hours of exposure to $10 \mu \mathrm{g} / \mathrm{mL}$ AgNP, ${ }^{15}$ whereas the current study demonstrated a decrease in the viability of hBMSC to the $70 \%$ viability threshold when $10 \mathrm{~nm}$ PVP-coated AgNP were used, which suggests that smaller diameter nanoparticles are more cytotoxic. Rosario et al. reported a viability reduction to $90 \%$ of control for MG-63 human osteosarcoma cells exposed to $10 \mu \mathrm{g} / \mathrm{mL} 10 \mathrm{~nm}$ PVP-coated AgNP exposure and reached the $70 \%$ viability threshold at $50 \mu \mathrm{g} / \mathrm{mL}$. ${ }^{16}$ The current study using primary stem cells exposed to $10 \mathrm{~nm}$ PVP-coated AgNP from the same supplier resulted in the $70 \%$ viability threshold at $10 \mu \mathrm{g} / \mathrm{mL}$. Similarly, a 24-hour exposure of immortalized human skin keratinocyte cells ${ }^{17}$ and MCF-7 breast cancer cells $^{18}$ to $10 \mu \mathrm{g} / \mathrm{mL}$ of uncoated $20 \mathrm{~nm} \mathrm{AgNP}$ resulted in cell viabilities of $67 \%$ and $76 \%$, respectively. Exposure of various cell types for 24 hours to $10 \mu \mathrm{g} / \mathrm{mL}$ of both PVP 
and uncoated $\mathrm{AgNP}$ was found to have no observed cytotoxic effect or meets the threshold as a potential cytotoxicant. These results support the findings in the current study that identify 1 and $5 \mu \mathrm{g} / \mathrm{mL} 10 \mathrm{~nm}$ PVP-coated AgNP concentrations as subcytotoxic and $10 \mu \mathrm{g} / \mathrm{mL}$ as a concentration with borderline cytotoxic potential.

Prolonged or permanent exposure to a medical device implant requires further evaluation beyond 24-hour cytotoxicity testing and includes, but is not limited to, longer term animals tests for systemic toxicity, skin sensitization and irritation, and implantation. In this study, in vitro exposure of hBMSC to AgNP up to 21 days revealed significant differences in proliferation and markers of osteogenic differentiation. Castiglioni et al. reported dose-dependent cytotoxicity to $35 \mathrm{~nm}$ uncoated AgNP with a decrease in Saos-2 osteoblast-like cell viability to $\sim 90 \%$ of control after a 5day exposure to 1 and $5 \mu \mathrm{g} / \mathrm{mL}$ and a similar decrease to $\sim 70 \%$ for $10 \mu \mathrm{g} / \mathrm{mL}$ exposures. ${ }^{19}$ In addition, these AgNP were not found to affect the differentiation of hBMSC into osteoblasts after 15 days in culture as judged by Alizarin Red staining of calcium deposits. Nanoparticle size plays a significant role in many in vitro endpoints except for DNA damage; exposed particle surface area was identified as a major factor influencing biological responses ${ }^{9}$ and could explain some of the discrepancies between these studies. Since $10 \mathrm{~nm} \mathrm{AgNP}$ has 3.5 times the surface area of $35 \mathrm{~nm} \mathrm{AgNP}$ per unit mass, the surface area of $5 \mu \mathrm{g} / \mathrm{mL}$ concentration of $10 \mathrm{~nm} \mathrm{AgNP}$ would therefore roughly corresponds to the surface area in $17.5 \mu \mathrm{g} / \mathrm{mL}$ of $35 \mathrm{~nm}$ AgNP; this is within the range of $10-25 \mu \mathrm{g} / \mathrm{mL} 35 \mathrm{~nm}$ AgNP used by Castiglioni et al. showing calcium deposition after 15 days. Continuous exposure to $5 \mu \mathrm{g} / \mathrm{mL}$ AgNP in this study resulted in cytotoxicity at the 7-day timepoint, and no evidence of osteogenic differentiation was observed; thus, factors in addition to the surface area appear to play a role in predicting toxicity.

One possible explanation is that particle size affects uptake and intracellular localization that can have locationdependent toxic effects. Uptake studies in human fibroblasts revealed that $30 \mathrm{~nm}$ gold nanoparticles are retained within the cytoplasm while $5 \mathrm{~nm}$ gold nanoparticles were found within the nucleus. ${ }^{20}$ Particle uptake studies in human mesenchymal stem cells exposed to $70 \mathrm{~nm}$ AgNP demonstrated perinuclear localization. ${ }^{21}$ Studies have shown that both uncoated $^{22}$ and PVP-coated $10 \mathrm{~nm} \mathrm{AgNP}{ }^{23}$ were also localized within lysosomes in multiple cell lines. Uptake of $10 \mathrm{~nm}$ PVP-coated AgNP in L929 fibroblasts exposed to $50 \mu \mathrm{g} / \mathrm{mL} \mathrm{AgNP}$ was found to be $5 \mu \mathrm{g} / \mathrm{cell}$ after 24 hours of exposure. ${ }^{23}$ Nanoscale ionic silver crystals were also found to form using TEM imaging and EDXS elemental analysis. Crystal formation was attributed to the dissolution of ionic silver from AgNP due to the acidic environment within lysosomes; silver ions could precipitate due to the presence of intracellular chloride and thiol-containing compounds.

Dissolution of silver ions from AgNP plays a major role in AgNP toxicity. Rosario et al. reported a greater cytotoxic response in MG-63 cells from $\mathrm{AgNO}_{3}$ compared with the corresponding mass of AgNP for exposures up to 48 hours but noted that by 7 days of exposure to $5 \mu \mathrm{g} / \mathrm{mL} 10 \mathrm{~nm}$ AgNP, MG-63 cells lost all proliferative capacity determined by the clonogenic assay while the corresponding $5 \mu \mathrm{g} / \mathrm{mL}$ of $\mathrm{Ag}^{+}$ions had significantly reduced proliferative capacity. ${ }^{16}$
In the present study, it is possible that cytotoxicity observed in the continuous exposure scenario could have been due to a larger contribution from intracellular particles compared with the single or repeated exposures. This conjecture is supported by the proliferation of hBMSC in the single and repeated exposures at $1 \mu \mathrm{g} / \mathrm{mL}$ being statistically different from the proliferation in the continuous exposure at day 14 for hBMSC cultured with osteogenic supplements. The trend appears to continue for day 21, although the difference between $1 \mu \mathrm{g} / \mathrm{mL}$ repeated and continuous exposures was not statistically significant.

Other studies support that the effect on AgNP on hBMSC proliferation could be partially attributed to early molecular initiating events, such as the dysregulation of genes related to cell metabolism or reactive oxygen species (ROS) defense, which lead to cytotoxicity. Genes related to osteogenesis were upregulated after 24-hour exposure to $20 \mu \mathrm{g} / \mathrm{mL}$ of $20 \mathrm{~nm}$ AgNP to the MC3T3-E1 mouse osteoblast cell line. ${ }^{24}$ Similarly, a decrease in proliferation in hBMSC and upregulation of markers related to endoplasmic reticulum and ROS stresses were found, but no changes to osteogenic differentiation markers were found in hBMSC. ${ }^{25}$ Calcium deposition can be considered a definitive marker of successful osteogenic differentiation; hBMSC exposure to 10 and $25 \mu \mathrm{g} / \mathrm{mL} 35 \mathrm{~nm}$ AgNP over 5 days in another study did not cause a change in calcium deposition. ${ }^{19}$ In contrast, ALP and mineralization in the current study were significantly impaired for most of the investigated concentrations and exposure scenarios. These effects occurred concomitantly with a marked decrease in proliferation, which may represent a cytotoxic response rather than impairment of differentiation.

Although repeated and continuous exposures at $5 \mu \mathrm{g} / \mathrm{mL}$ or higher prevented deposition of hydroxyapatite, this marker was not affected under the single exposure scenario and may hold promise as an antimicrobial in orthopedic surgeries. Prophylactic use of antibiotics in orthopedic surgeries includes a single preoperative dose and a possible re-dosing 24 or 48 hours after the operation. ${ }^{26,27}$ In a similar manner to prophylactic antibiotic use, Gao et al. demonstrated that the majority of silver from $70 \mathrm{~nm}$ AgNP on the surface of a PEEK implant were released within 24 hours, yielding a $5 \mu \mathrm{g} / \mathrm{mL}$ extract; the cumulative silver release curve plateaued after 5 days. ${ }^{28}$ A reduction in viability of MC3T3E1 osteoblast precursor cells was observed, but this difference versus the control was eliminated by day 5 due to dilution of the silver with repeated media changes. Implantation of this material into rats demonstrated antimicrobial activity and was observed to osseointegrate similar to the materials without AgNP after 2 weeks of implantation.

\section{Conclusions}

The present study examines the effect of different exposure scenarios on the proliferation and osteocyte function of differentiated hBMSC. Notably, a cytotoxic threshold of $10 \mu \mathrm{g} / \mathrm{mL}$ PVP-coated $10 \mathrm{~nm}$ AgNP for hBMSC was determined, but this single 24-hour exposure caused a loss of osteocyte function in the 21-day culture. The viability of hBMSC exposed to $5 \mu \mathrm{g} / \mathrm{mL}$ was not significantly different from controls in the 24-hour exposure, but this single 24-hour exposure caused a delay in the observation of ALP. This marker and 
subsequent mineralization were not observed after repeated 24-hour exposures at the $5 \mu \mathrm{g} / \mathrm{mL}$ concentration. hBMSC exposed to the $1 \mu \mathrm{g} / \mathrm{mL} \mathrm{AgNP}$ concentration were observed to express ALP and deposit hydroxyapatite in all exposure scenarios, but continuous exposure caused significantly lower proliferation rates than the other two exposure scenarios at day 14. Differences in proliferation, ALP expression, and hydroxyapatite deposition were detected in the different exposure scenarios at AgNP concentrations identified as subcytotoxic in a 24-hour exposure assay.

The well-known ability of silver to prevent the growth of a broad spectrum of infectious organisms without the use of antibiotics makes it an attractive additive to medical device implants. However, AgNP exposure at sufficient concentrations and/or exposure durations does have an adverse effect on hBMSC osteogenic differentiation and subsequent function as osteocytes. AgNP-containing medical device materials must be carefully formulated and evaluated to determine the benefit of infection control versus the risk of adverse effects on bone marrow. ${ }^{29}$

Evaluating nanomaterials with biomedical applications presents challenges due to the vast number of nanoparticle physical and chemical parameters (e.g., size, shape, and coating) that influence biological and toxicological effects. For example, assumptions about the safety and performance of AgNP might not be supportable if there is a change in one or more of these parameters. Due to the vast number of nanomaterials with varying sizes, shapes, surface coatings and chemistries, and routes of exposure, in vitro human stem cell models that offer prolonged exposure scenarios compared with traditional in vitro models represent a potentially valuable component of integrated test strategies to evaluate nanomaterials.

\section{Acknowledgments}

The authors acknowledge Brendan Casey and the CDRH Advanced Characterization Facility for TEM imaging of the AgNP. This study was supported by funds from the U.S. Food and Drug Administration.

\section{Disclaimer}

The findings and conclusions in this article have not been formally disseminated by the Food and Drug Administration and should not be construed to represent any agency determination or policy. The mention of commercial products, their sources, or their use in connection with material reported herein is not to be construed as either an actual or implied endorsement of such products by the Department of Health and Human Services.

\section{Author Disclosure Statement}

No competing financial interests exist.

\section{References}

1. Chua HS, Whitehouse SL, Lorimer M, et al. Mortality and implant survival with simultaneous and staged bilateral total knee arthroplasty experience from the Australian Orthopaedic Association National Joint Replacement Registry. J Arthroplasty 2018:33;3167-3173.
2. Al-Mulhim FA, Baragbah MA, Sadat-Ali M, et al. Prevalence of surgical site infection in orthopedic surgery: A 5year analysis. Int Surg 2014:99;264-268.

3. Goel A, Meher MK, Gupta P, et al. Microwave assisted $\kappa$-carrageenan capped silver nanocomposites for eradication of bacterial biofilms. Carbohyd Polym 2019:206;854-862.

4. Khalid HF, Tehseen B, Sarwar Y, et al. Biosurfactant coated silver and iron oxide nanoparticles with enhanced antibiofilm and anti-adhesive properties. J Hazard Mater 2019:364;441-448.

5. Liu M, Liu T, Chen X, et al. Nano-silver-incorporated biomimetic polydopamine coating on a thermoplastic polyurethane porous nanocomposite as an efficient antibacterial wound dressing. J Nanobiotechnol 2018:16;89.

6. Chen Y, Dan N, Dan W, et al. A novel antibacterial acellular porcine dermal matrix cross-linked with oxidized chitosan oligosaccharide and modified by in situ synthesis of silver nanoparticles for wound healing applications. Mater Sci Eng C Mater Biol Appl 2019:94;1020-1036.

7. Choi J, Reipa V, Hitchins VM, et al. Physicochemical characterization and in vitro hemolysis evaluation of silver nanoparticles. Toxicol Sci 2011:123;133-143.

8. Krajewski S, Prucek R, Panacek A, et al. Hemocompatibility evaluation of different silver nanoparticle concentrations employing a modified Chandler-loop in vitro assay on human blood. Acta Biomater 2013:9;7460-7468.

9. Ahmed KBR, Nagy AM, Brown RP, et al. Silver nanoparticles: Significance of physicochemical properties and assay interference on the interpretation of in vitro cytotoxicity studies. Toxicol In Vitro 2017:38;179-192.

10. Akter M, Sikder MT, Rahman MM, et al. A systematic review on silver nanoparticles-induced cytotoxicity: Physicochemical properties and perspectives. J Adv Res 2018:9;116.

11. Bar-Ilan O, Albrecht RM, Fako VE, et al. Toxicity assessments of multisized gold and silver nanoparticles in zebrafish embryos. Small 2009:5;1897-1910.

12. International Organization for Standardization. Biological Evaluation of medical devices. (ISO Standard No. 10993: 2009). https://www.iso.org/standard/36406.html (last accessed Apr. 27, 2012).

13. Sussman EM, Jayanti P, Dair BJ, et al. Assessment of total silver and silver nanoparticle extraction from medical devices. Food Chem Toxicol 2015:85;10-19.

14. De Matteis V, Cascione M, Toma CC, et al. Silver nanoparticles: Synthetic routes, in vitro toxicity and theranostic applications for cancer disease. Nanomaterials (Basel) 2018:8;319.

15. Sengstock C, Diendorf J, Epple M, et al. Effect of silver nanoparticles on human mesenchymal stem cell differentiation. Beilstein J Nanotechnol 2014:5;2058-2069.

16. Rosario F, Hoet P, Santos C, et al. Death and cell cycle progression are differently conditioned by the AgNP size in osteoblast-like cells. Toxicology 2016:368;103-115.

17. Kreeinthong S, Uawithya P. Effects of Short-term silver nanoparticle exposure on proliferative signaling pathway in human skin keratinocyte. J Physiol Biomed Sci 2014: 27;48-53.

18. Çiftçi H, Türk M, Tamer U, et al. Silver nanoparticles: Cytotoxic, apoptotic, and necrotic effects on MCF-7 cells. Turkish J Biol 2013:37;573-581.

19. Castiglioni S, Cazzaniga A, Locatelli L, et al. Silver nanoparticles in orthopedic applications: New insights on their 
effects on osteogenic cells. Nanomaterials (Basel) 2017:7; 124.

20. Berry CC, de la Fuente JM, Mullin M, et al. Nuclear localization of HIV-1 tat functionalized gold nanoparticles. IEEE Trans Nanobioscience 2007:6;262-269.

21. Ahlberg S, Antonopulos A, Diendorf J, et al. PVP-coated, negatively charged silver nanoparticles: A multi-center study of their physicochemical characteristics, cell culture and in vivo experiments. Beilstein J Nanotechnol 2014:5; 1944-1965.

22. Hsiao I-L, Hsieh Y-K, Chuang C-Y, et al. Effects of silver nanoparticles on the interactions of neuron- and glia-like cells: Toxicity, uptake mechanisms, and lysosomal tracking. Environ Toxicol 2017:32;1742-1753.

23. Wildt BE, Celedon A, Maurer EI, et al. Intracellular accumulation and dissolution of silver nanoparticles in L-929 fibroblast cells using live cell time-lapse microscopy. Nanotoxicology 2016:10;710-719.

24. Qing T, Mahmood M, Zheng Y, et al. A genomic characterization of the influence of silver nanoparticles on bone differentiation in MC3T3-E1 cells. J Appl Toxicol 2018:38;172-179.

25. Pauksch L, Hartmann S, Rohnke M, et al. Biocompatibility of silver nanoparticles and silver ions in primary human mesenchymal stem cells and osteoblasts. Acta Biomater 2014:10;439-449.

26. Backes M, Dingemans SA, Dijkgraaf MGW, et al. Effect of antibiotic prophylaxis on surgical site infections following removal of orthopedic implants used for treatment of foot, ankle, and lower leg fractures: A Randomized Clinical Trial. JAMA 2017:318;2438-2445.

27. Gans I, Jain A, Sirisreetreerux N, et al. Current practice of antibiotic prophylaxis for surgical fixation of closed long bone fractures: A survey of 297 members of the Orthopaedic Trauma Association. Patient Saf Surg 2017:11;2.

28. Gao C, Wang Y, Han F, et al. Antibacterial activity and osseointegration of silver-coated poly(ether ether ketone) prepared using the polydopamine-assisted deposition technique. J Mater Chem B 2017:5;9326-9336.

29. Petrochenko PE, Zheng J, Casey BJ, et al. NanosilverPMMA composite coating optimized to provide robust antibacterial efficacy while minimizing human bone marrow stromal cell toxicity. Toxicol In Vitro 2017:44;248-255.

Address correspondence to: Dr. Peter L. Goering Office of Science and Engineering Laboratories Center for Devices and Radiological Health US Food and Drug Administration 10903 New Hampshire Avenue Silver Spring, MD 20993

E-mail: peter.goering@fda.hhs.gov 